\title{
Article \\ The Influence of Maximal Strength and Knee Angle on the Reliability of Peak Force in the Isometric Squat
}

\author{
Arthur E. Lynch ${ }^{1}$, Robert W. Davies ${ }^{1}{ }^{1}$, Philip M. Jakeman ${ }^{1}{ }^{1}$, Tim Locke ${ }^{1}$, Joanna M. Allardyce ${ }^{1,2}$ \\ and Brian P. Carson 1,2,*iD \\ 1 Department of Physical Education and Sport Sciences, Faculty of Education and Health Sciences, \\ University of Limerick, V94 T9PX Limerick, Ireland; Arthur@sigmanutrition.com (A.E.L.); \\ robert.davies@ul.ie (R.W.D.); phil.jakeman@ul.ie (P.M.J.); Tim.locke123@hotmail.com (T.L.); \\ joanna.allardyce@ul.ie (J.M.A.) \\ 2 Health Research Institute, University of Limerick, V94 T9PX Limerick, Ireland \\ * Correspondence: Brian.Carson@ul.ie; Tel.: +353-(0)61-234943
}

Citation: Lynch, A.E.; Davies, R.W.; Jakeman, P.M.; Locke, T.; Allardyce, J.M.; Carson, B.P. The Influence of Maximal Strength and Knee Angle on the Reliability of Peak Force in the Isometric Squat. Sports 2021, 9, 140. https://doi.org/10.3390/ sports 9100140

Academic Editor: Kevin Lamb

Received: 20 July 2021

Accepted: 2 October 2021

Published: 9 October 2021

Publisher's Note: MDPI stays neutral with regard to jurisdictional claims in published maps and institutional affiliations.

Copyright: (c) 2021 by the authors. Licensee MDPI, Basel, Switzerland. This article is an open access article distributed under the terms and conditions of the Creative Commons Attribution (CC BY) license (https:// creativecommons.org/licenses/by/ $4.0 /)$.

\begin{abstract}
This study aimed to investigate the test-retest reliability of peak force in the isometric squat across the strength spectrum using coefficient of variation $(\mathrm{CV})$ and intra-class correlation coefficient (ICC). On two separate days, 59 healthy men (mean (SD) age 23.0 (4.1) years; height $1.79(0.7) \mathrm{m}$; body mass $84.0(15.2) \mathrm{kg}$ ) performed three maximal effort isometric squats in two positions (at a $120^{\circ}$ and a $90^{\circ}$ knee angle). Acceptable reliability was observed at both the $120^{\circ}$ ( CV = 7.5 (6.7), ICC $=0.960[0.933,0.977])$ and $90^{\circ}$ positions $(\mathrm{CV}=9.2(8.8), \mathrm{ICC}=0.920[0.865,0.953])$. There was no relationship between peak force in the isometric squat and the test-retest reliability at either the $120^{\circ}(\mathrm{r}=0.052, p=0.327)$ or $90^{\circ}(\mathrm{r}=0.014, p=0.613)$ positions. A subgroup of subjects $(n=17)$ also completed the isometric squat test at a $65^{\circ}$ knee angle. Acceptable reliability was observed in this position $(\mathrm{CV}=9.6(9.3), \mathrm{ICC}=0.916[0.766,0.970])$ and reliability was comparable to the $120^{\circ}$ and $90^{\circ}$ positions. Therefore, we deem isometric squat peak force output to be a valid and reliable measure across the strength spectrum and in different isometric squat positions.
\end{abstract}

Keywords: muscle strength; isometric contraction; measurement; reproducibility of results; strength testing

\section{Introduction}

Assessments of maximal voluntary force (or strength) can provide valuable insight into one's physical condition, differentiate strength characteristics between individuals, evaluate the efficacy of a training intervention, as well as inform exercise load prescription for future training programs [1]. However, there are several variables that can confound maximal force measurement (e.g., joint angle, angular velocity, use of the stretch-shortening cycle, and other skill-related components of the test). The use of isokinetic dynamometry can control for most of these confounding variables [1]. However, the external validity of these isolated, single-joint assessments in certain applications is low. Multi-articular assessments may be preferable to single-joint dynamometry, particularly for practitioners [2-4].

Traditional multi-articular measures of lower-body maximal strength such as the one-repetition maximum (1RM) back squat have much greater external validity compared to dynamometry-based assessments of strength [1]. However, such assessments may be unsuitable in certain circumstances due to both practical and methodological issues surrounding the conduct of 1RM back squat testing; in particular the control of the range of motion and the reliability of the test in certain populations [5,6]. The latter is highlighted by the susceptibility of 1RM to learning effects [6]. Furthermore, the reliability of 1RM testing tends to improve with training experience and/or baseline strength level [6]. Due to unfamiliarity with the exercise, it is unlikely that a true indication of muscle force output can be distinguished from the skill-related aspects of a 1RM test in untrained individuals. 
Moreover, 1RM testing can be quite demanding and poses a level of risk that could be deemed unacceptable [7]. For athletes, this may be a concern as repeated exposure to high loads may increase injury risk and/or residual fatigue [8,9].

The isometric squat (ISq) presents a credible alternative to the $1 \mathrm{RM}$ back squat as it is conducted at a fixed position, with acceptable levels of test-retest reliability for peak force $(<10 \% \mathrm{CV}, \geq 0.8 \mathrm{ICC})[10,11]$. The ISq test is less demanding than the $1 \mathrm{RM}$, suggesting the ISq should provide good test-retest reliability across the strength spectrum. Herein, we operationally define the strength spectrum as covering the broad range of strength levels from untrained individuals up to and including highly strength-trained individuals. However, to the best of our knowledge, no previous study has investigated the reliability of peak force (PF) in the ISq across the strength spectrum.

PF in the ISq is commonly assessed at knee angles of $120^{\circ}$ and $/$ or $90^{\circ}$, where full knee extension is $180^{\circ}[12,13]$. The $120^{\circ}$ knee angle position (ISq120) closely replicates the strongest position of the back squat [14], whereas the $90^{\circ}$ knee angle position (ISq90) approximates the 'sticking point' of the back squat (i.e., the point at which momentary muscular failure occurs during the lift) [11,15]. Although it produces lower peak forces compared to ISq ${ }_{120}$, the ISq $q_{90}$ correlates more strongly $(\mathrm{r}=0.70-0.86)$ with dynamic $1 \mathrm{RM}$ back squat performance $[10,11,16]$. Whilst both test positions have been shown to produce reliable data for PF $[10,11,16,17]$, previous ISq investigations have not explored the reliability of ISq positions that require knee angles of $<90^{\circ}$ [13]. Reasons for this may include difficulty attaining the position and/or lower PF reliability. Palmer et al. (2017) found isometric squat reliability reduced as the knee angle at which the test was performed lowered (i.e., $\mathrm{CV}$ at $150^{\circ},<120^{\circ},<90^{\circ}$ ), which may indicate that the reliability of the isometric squat at a knee angle of $<90^{\circ}$ may be inferior to that of higher knee angles [17]. Beyond this, it is difficult to speculate as, to the authors' knowledge no data exist documenting the reliability of the isometric squat at a knee angle of $<90^{\circ}$. However, it could be argued that in populations unfamiliar with deeper squatting positions (i.e., $<90^{\circ}$ ), the ISq assessment may be safer compared to typical isoinertial assessments that demand the same range of motion (e.g., 1RM back squat). Additionally, assessment of strength in deeper squatting positions may be of relevance to athletes, particularly those involved in strength sports like (e.g., powerlifting and Olympic weightlifting) who regularly squat to knee angles of $<90^{\circ}$ [18].

The aim of the present study was twofold. Firstly, we aimed to investigate the relationship between ISq strength (as indicated by PF in the ISq), and test-retest reliability of PF in the ISq. It was a priori hypothesized that ISq reliability would be influenced by maximal ISq strength. Secondly, we sought to compare the reliability of PF in the ISq at a deeper squat position, which approximates the criteria for full depth in the back squat exercise (i.e., a $65^{\circ}$ knee angle [ $\left.\mathrm{ISq}_{65}\right]$ ] [14,19-21]. We hypothesized that test-retest reliability of PF in the ISq would be correlated with baseline ISq strength and that both PF output in the ISq and test-retest reliability would be greater as the knee angle moved closer to full extension (i.e., $\mathrm{ISq}_{120}>\mathrm{ISq}_{90}>\mathrm{ISq}_{65}$ ).

\section{Materials and Methods}

\subsection{Subjects}

Prior to inclusion subjects were informed of the benefits and risks of participation before providing written informed consent. Eligibility criteria were: (i) men, (ii) 18 to 35 years of age, (iii) habitually active and in good general health with no current injuries, illness, or history of disease. In total, 59 men (mean (SD) age 23.5 (4.1) years, body mass 84.1 $(15.2) \mathrm{kg}$, height $1.8(0.7) \mathrm{m}$ ) were recruited from the local area and voluntarily took part in the study. Subjects' training age ranged from 0 to 13 years; with relative 1RM back squat strength ranging from 0.7 to $2.7 \mathrm{~kg} \cdot \mathrm{kg}^{-1}$ body mass. The sample consisted of 8 untrained subjects (no prior strength training experience whatsoever), 42 moderately strength-trained subjects (i.e., $\geq 6$ months strength training experience, $1 \mathrm{RM}<1.5 \mathrm{~kg} \cdot \mathrm{kg}^{-1}$ body mass), and 9 highly strength-trained subjects (competitive powerlifters, $\geq 3$ years strength training 
experience, $1 \mathrm{RM} \geq 2.0 \mathrm{~kg} \cdot \mathrm{kg}^{-1}$ body mass). A sub-group of 17 subjects (mean (SD) age $22.6(2.6)$ years, body mass $81.9(11.3) \mathrm{kg}$, height $1.8(0.7) \mathrm{m})$ performed the ISq in a third position, at a $65^{\circ}$ knee angle $\left(\mathrm{ISq}_{65}\right)$.

\subsection{Instrumentation}

ISq testing was conducted using a custom-made ISq rack (Odin Gym Equipment, Ireland) with a fixed barbell, adjustable in height, positioned above two force plates (AMTI, Watertown, MA, USA). The rack was bolted to the floor around the force plates (see Figure 1). Cortex motion analysis software (Rohnert Park, CA, USA) was used for the collection of PF data. For each contraction, ground reaction force data were sampled at $1 \mathrm{kHz}$ and $\mathrm{PF}$ was defined as the highest value recorded from the force-time curve (excluding subject body weight).
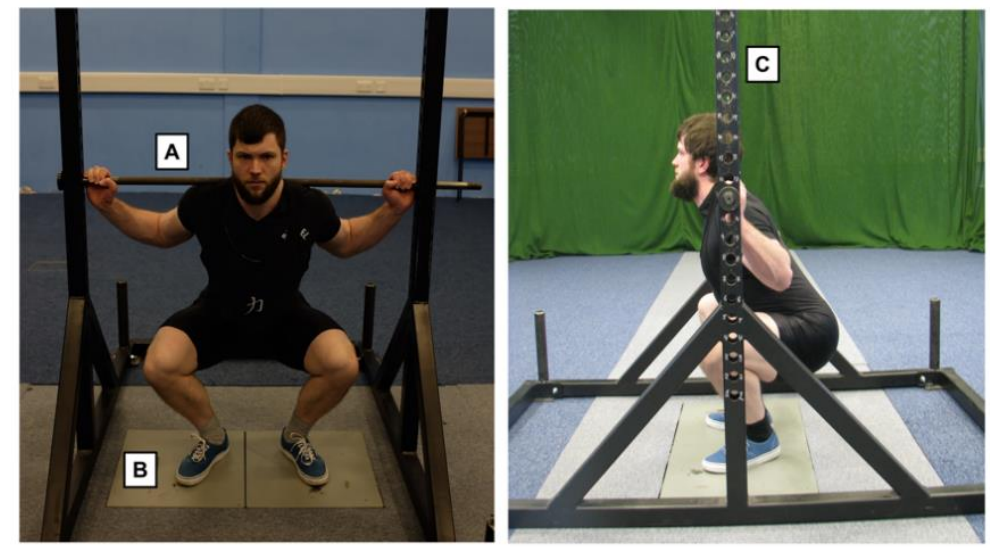

Figure 1. Isometric squat rack apparatus and subject setup: $($ left $)=$ front view, $($ right $)=$ side view. $\mathrm{A}=$ fixed barbell, $\mathrm{B}=\mathrm{AMTI}$ force plates, $\mathrm{C}=$ adjustable bar heights $(2.5 \mathrm{~cm}$ between rack heights). In this example, the isometric squat was performed with knee and hip angles of $65^{\circ}$ and $115^{\circ}$, respectively.

\subsection{Procedures}

Subjects reported to the lab at the same time (10:00 to 14:00) each test day to minimize diurnal variation. Eligibility and familiarization with the test procedures was undertaken 24 to $72 \mathrm{~h}$ prior to starting the study. Subjects were instructed to maintain their normal eating habits throughout the study. Dietary intake was recorded prior to the first test day and each subject was instructed to repeat this intake before the second test day. Subjects were instructed to refrain from any formal lower body exercise $48 \mathrm{~h}$ prior to testing and instructed to maintain their normal eating habits throughout the study.

The subjects removed their shoes for the duration of the test procedures to control for any variation in footwear between subjects or between test sessions. Prior to commencing the warm-up, the rack heights that corresponded to the required ISq test positions were obtained. To do this, the subject assumed their preferred squatting position (i.e., stance width and foot position) on top of the force plates. The distance between the feet at the anterior (i.e., distance between the two first distal phalanges) and posterior extremities (i.e., distance between the most posterior and medial aspect of the left and right calcaneus bones) was measured, recorded, and marked with tape. For repeat trials, tape was re-laid using the measurements recorded in the first testing session, allowing for consistency across trials. Whilst maintaining this same squatting stance, each subject was then instructed to descend until the desired knee angle (i.e., $120^{\circ}, 90^{\circ}$, or $65^{\circ}$ ) was obtained. The knee angle was measured using a plastic goniometer (Fabrication Enterprises, White Plains, NY, USA) which was placed on the lateral condyle of the femur, the fixed end was aligned with the greater trochanter of the femur, and the moving arm aligned with the lateral malleolus 
of the ankle. Additionally, angles at the hip and ankle joints were recorded to ensure within-subject consistency across trials.

The testing order for the two positions ( $\mathrm{ISq}_{120}$ and $\mathrm{ISq}_{90}$ ) was randomized for each subject on the first test day and this same order was repeated on the second test day. Subjects completed three warm-up ISq contractions in the first test position prior to measurement (50\%, $70 \%$, and $90 \%$ of perceived maximal effort) which were maintained for $3 \mathrm{~s}$ with a $1 \mathrm{~min}$ rest provided between each sub-maximal contraction. Immediately following the sub-maximal contractions, a 3 min rest period was provided before the first maximal effort contraction and then again for each subsequent maximal effort contraction [10]. Subjects were given standard verbal encouragement from the investigators, who instructed subjects to "push as hard and fast as possible into the bar" and to maintain peak force output for the duration of the $4 \mathrm{~s}$ contraction. Three maximal effort ISq contractions were performed in each position. Vertical ground reaction force data were sampled at $1 \mathrm{kHz}$ and excluded if any countermovement was evident. PF was determined as the highest value attained out of the three attempts on each test day. All subjects performed three maximal effort ISq contractions at $\mathrm{ISq}_{120}$ and $\mathrm{ISq}_{90}$ on two separate test days at least $72 \mathrm{~h}$ apart (range: 72-168 h). A minimum of $72 \mathrm{~h}$ rest was provided between test days, which has been evidenced to allow full recovery from strenuous lower body dominant resistance exercise [22]. A sub-group of 17 subjects also performed an additional three maximal effort ISq contractions at ISq $\mathrm{q}_{65}$ on each test day.

\subsection{Statistical Analyses}

Test-retest (i.e., day 1 vs. day 2) reliability scores were calculated for PF for all subjects, analyzed as one group $(n=59)$. Normality and homogeneity of variance were assessed prior to analysis (Shapiro Wilk and Levene's test respectively). A two-way random model with absolute agreement was used to calculate the intra-class correlation coefficient (ICC) [23]. An ICC over 0.9 was defined as highly reliable, between 0.8 and 0.9 as moderately reliable, and below 0.8 as not reliable [23-25].

The between-day coefficient of variation (CV) was calculated. A CV of $<10 \%$ is accepted as reliable [6]. Paired samples $t$-tests were used to assess differences between days (day 1 vs. day 2) for PF and between positions ( $\mathrm{ISq}_{120}$ vs. $\mathrm{ISq}_{90}$ ) for both PF and $\mathrm{CV}$, with the alpha level set at $p<0.05$. To assess the relationship between maximal ISq strength and the reliability of PF measurement, a correlation analysis (Pearson's correlation coefficient) was conducted in Microsoft Excel by plotting subjects' PF scores at ISq $q_{120}$ and ISq ${ }_{90}$ against their respective $\mathrm{CV}$ scores in each position.

To assess the influence of knee angle on PF, a one-way ANOVA was performed to compare differences in PF between positions ( $\mathrm{ISq}_{120}$ vs. ISq ${ }_{90}$ vs. ISq $\mathrm{I}_{65}$ ), with the alpha level set at $p<0.05$. Normality and homogeneity of variance were assessed prior to this analysis. This same analysis was also performed with subjects divided into their respective sub-groups based on training experience (i.e., untrained vs. moderately trained vs. highly trained. To assess the influence of knee angle on ISq reliability, a one-way ANOVA was performed to compare differences in $\mathrm{CV}$ between positions. Where significant differences were detected, paired sample $t$-tests were used to identify differences between knee angles. Effect size $(d)$ was calculated for differences in PF between positions by dividing the position difference by the pooled standard deviation [25]. The effect sizes were classified as small (0.2), medium (0.5), or large (0.8) [26]. The between-day difference with upper and lower $(95 \%)$ levels of agreement (LOA) was used to assess systematic bias. Typical error (TE) was calculated for PF by dividing the standard deviation of the within- subject differences in PF between the two test days (i.e., the peak of day 1 and the peak of day 2 ) by $\sqrt{ } 2$ [27]. TE provides an indication of the change score required between trials to be considered "real" (i.e., outside the normal range of error associated with the test) [27]. As such, the TE could be considered an index of the sensitivity of the measure to detect change over time and provides real value for practitioners who may consider using the ISq to measure performance changes in response to a training program [11,27]. 


\section{Results}

\subsection{Relationship between Isometric Squat Strength and Measurement Reliability}

Reliability data for all subjects $(n=59)$ are presented in Table 1 . PF was significantly greater at $\mathrm{ISq}_{120}$ than at $\mathrm{ISq}_{90}(p<0.001)$. All variables achieved a high/acceptable level of reliability (ICC $>0.9 ; \mathrm{CV}<10 \%$ ) at both $\mathrm{ISq}_{120}$ and ISq $\mathrm{I}_{90}$ (Table 1). Furthermore, when the analysis was performed on the subject sub-groups (untrained vs. moderately trained vs. highly trained), no significant differences in measurement reliability were observed in either position $(p \geq 0.146)$. Correlation analyses of the test-retest reliability revealed no relationship between maximal ISq strength and ISq reliability at either ISq $\mathrm{I}_{120}(\mathrm{r}=0.052$, $p=0.327)$ or $\mathrm{ISq}_{90}(\mathrm{r}=0.014, p=0.613)$ as shown in Figure 2 .

Table 1. Reliability of peak isometric force at $\mathrm{ISq}_{120}$ and $\mathrm{ISq}_{90}(n=59)$.

\begin{tabular}{|c|c|c|c|c|}
\hline \multicolumn{5}{|c|}{ Peak Force (N) } \\
\hline & Day 1 [Mean (SD)] & Day 2 [Mean (SD)] & \multicolumn{2}{|c|}{$\begin{array}{c}\text { Difference (Day 2-Day 1) } \\
\text { Mean [LLOA, ULOA] }\end{array}$} \\
\hline $\mathrm{ISq}_{120}$ & $1579(536)$ & $1536(567)$ & \multicolumn{2}{|c|}{$-44[-100,12]$} \\
\hline $\mathrm{ISq}_{90}$ & $1035(298)^{a}$ & $958(275)^{a}$ & \multicolumn{2}{|c|}{$-73[-114,-32]$} \\
\hline \multicolumn{5}{|c|}{ Test-Retest Reliability } \\
\hline & CV [Mean (SD)] & $\mathrm{ICC}[95 \% \mathrm{CI}]$ & TE (N) & TE (\%) \\
\hline $\mathrm{ISq}_{120}$ & $7.5(6.7)$ & $0.960[0.933,0.977]$ & 140.2 & 9.5 \\
\hline $\mathrm{ISq}_{90}$ & $9.2(8.8)$ & $0.920[0.865,0.953]$ & 82.6 & 8.8 \\
\hline
\end{tabular}

LLOA $=$ Lower limits of agreement, ULOA = Upper limits of agreement, CV = coefficient of variation, ICC $=$ intraclass correlation coefficient, $\mathrm{TE}=$ Typical error, $95 \% \mathrm{CI}=95 \%$ confidence interval, a $=$ significantly different peak isometric force from the ISq 120 position $(p<0.001)$.
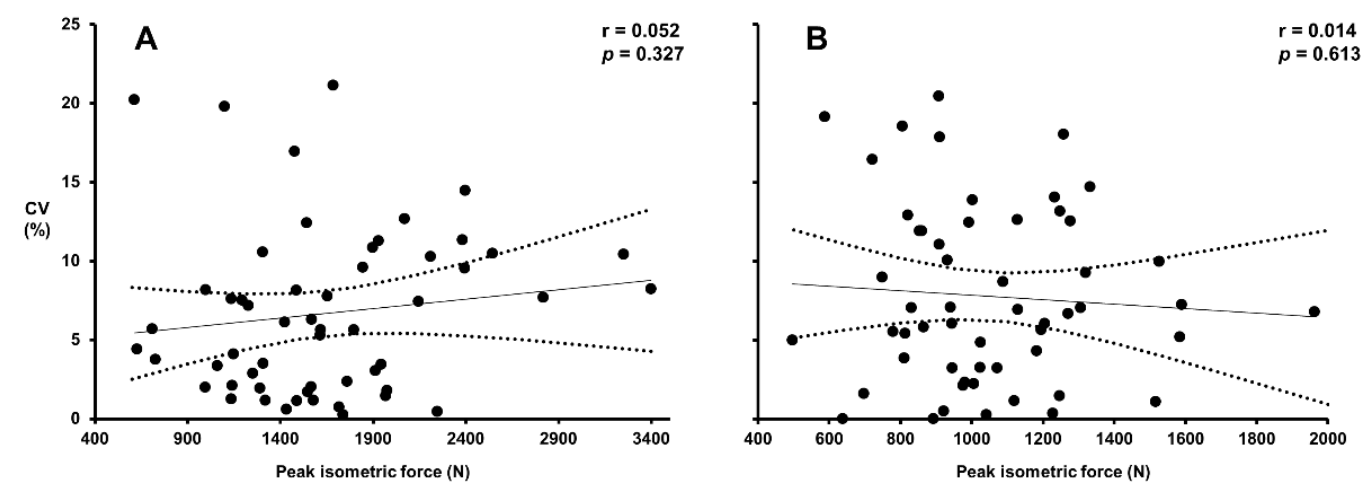

Figure 2. Correlation analyses of maximal ISq strength $(\mathrm{PF})$ and reliability via $\mathrm{CV}$ in the isometric squat: (A) represents the reliability of PF (highest values of day 1 and day 2) at ISq120, plotted against the corresponding CV values. (B) Represents the reliability of PF (highest values of day 1 and day 2) at ISq90, plotted against the corresponding CV values. Dashed lines indicate the $95 \%$ confidence intervals.

\subsection{Relationship between Isometric Squat Position and Measurement Reliability}

The test-retest reliability of PF measurement at $\mathrm{ISq}_{120}, \mathrm{ISq}_{90}$ and $\mathrm{ISq}_{65}$ in the subject sub-group $(n=17)$ is presented in Table 2. PF was significantly greater at $\mathrm{ISq}_{120}$ compared to $\mathrm{ISq}_{90}(p<0.001, d=0.8)$ and $\mathrm{ISq}_{65}(p<0.001, d=1.0)$. There was no significant difference in PF between the ISq 90 and $\mathrm{ISq}_{65}$ positions $(p=0.052, d=0.3)$. Results of the one-way ANOVA revealed that test-retest reliability was not different between positions $\left(\mathrm{ISq}_{120} \mathrm{CV}=7.3 \%\right.$; $\mathrm{ISq}_{90} \mathrm{CV}=8.9 \%$; $\mathrm{ISq}_{65} \mathrm{CV}=9.6 \%, p=0.6$ ) and an acceptable level of reliability was observed in all positions based on ICC values (ISq120 ICC = 0.969; $\mathrm{ISq}_{90} \mathrm{ICC}=0.892 ; \mathrm{ISq}_{65} \mathrm{ICC}=0.916$ ) Mean between-day difference (day 2-day 1 ) was determined via Bland-Altman analysis for PF in each position (Figure 3). No systematic bias was observed between test days for any variable or position (Figure 3). Finally, the 
TE values were determined for PF in each position $\left(\mathrm{ISq}_{120}=118 \mathrm{~N}(10.5 \%) ; \mathrm{ISq}_{90}=117 \mathrm{~N}\right.$ $(14.2 \%) ; \mathrm{ISq}_{65}=83 \mathrm{~N}(11.0 \%)$, see Table 2$)$.

Table 2. Reliability of peak isometric force across isometric squat positions $(n=17)$.

\begin{tabular}{|c|c|c|c|c|}
\hline \multicolumn{5}{|c|}{ Peak Force (N) } \\
\hline & Day 1 [Mean (SD)] & Day 2 [Mean (SD)] & \multicolumn{2}{|c|}{$\begin{array}{c}\text { Difference (Day 2-Day 1) } \\
\text { Mean [LLOA, ULOA] }\end{array}$} \\
\hline $\mathrm{ISq}_{120}$ & $1259(525)_{a, b}$ & $1197(467)_{a, b}$ & \multicolumn{2}{|c|}{$-63[-142,17]$} \\
\hline $\mathrm{ISq}_{90}$ & $919(287)_{\mathrm{c}, \mathrm{b}}^{\mathrm{a}, \mathrm{b}}$ & $848(288)_{c}$ & \multicolumn{2}{|c|}{$-50[-120,21]$} \\
\hline $\mathrm{ISq}_{65}$ & $820(205)_{c, a}$ & $826(233)_{c}$ & \multicolumn{2}{|c|}{$6[-53,66]$} \\
\hline \multicolumn{5}{|c|}{ Test-Retest Reliability } \\
\hline & CV [Mean (SD)] & ICC $[95 \% \mathrm{CI}]$ & TE (N) & TE (\%) \\
\hline $\mathrm{ISq}_{120}$ & $7.3(4.3)$ & $0.969[0.914,0.989]$ & 118 & 10.5 \\
\hline $\mathrm{ISq}_{90}$ & $8.9(6.7)$ & $0.892[0.702,0.961]$ & 117 & 14.2 \\
\hline $\mathrm{ISq}_{65}$ & $9.6(9.3)$ & $0.916[0.766,0.970]$ & 83 & 11.0 \\
\hline
\end{tabular}

LLOA = Lower limits of agreement, ULOA = Upper limits of agreement, CV = Coefficient of variation, ICC $=$ Intraclass correlation coefficient, $95 \% \mathrm{CI}=95 \%$ confidence interval, TE = Typical error, a = significantly different $(p<0.05)$ from ISq $q_{90}, \mathrm{~b}=$ significantly different from ISq $\mathrm{q}_{65}, \mathrm{c}=$ significantly different from ISq $\mathrm{q}_{120}$.

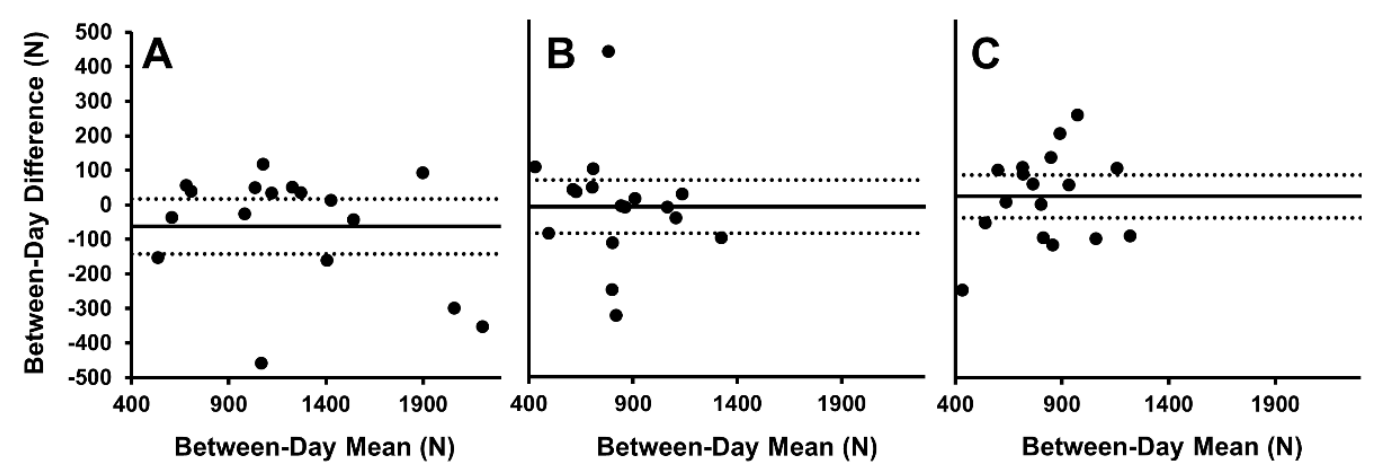

Figure 3. Bland-Altman plots for between-day peak isometric force. (A) PF at $\mathrm{ISq}_{120}$, (B) PF at ISq90, and (C) $\mathrm{PF}$ at $\mathrm{ISq}_{65}$. Solid line represents the mean difference; dashed lines represent $95 \%$ limits of agreement.

\section{Discussion}

Training status and by association strength has previously been shown to influence the reliability of strength measures such as 1RM squat and leg press, with greater reliability typically observed with increased strength and/or strength training experience [6,28,29]. Though previous literature has documented the reliability of a variety of ISq tests across populations with different levels of strength, no previous study has investigated the relationship between maximal ISq strength and the reliability of the measure $[10,11,16,17]$. Therefore, the initial aim of this study was to investigate the test-retest reliability of the ISq across the strength spectrum. Here we report acceptable levels of reliability for PF in a sample of 59 subjects (Table 1 ) based on previously established reliability cut-offs (i.e., $>0.8$ ICC and $<10 \%$ CV $[6,24,30]$. Correlation analyses revealed no relationship between maximal ISq strength (as indicated by PF) and ISq reliability at ISq 120 and ISq $q_{90}$ (Figure 2). It is worth noting however that based on Figure 2, all outliers (i.e., CV $\geq 15 \%$ ) seem to occur amongst individuals with lower peak force values.

Though no previous studies have directly investigated the relationship between maximal ISq strength and the reliability of ISq measurement, ISq reliability studies across different populations with distinct levels of strength are available to place the findings of this study in context. Tillin et al. (2013) is the only available study that measured ISq force variables in two distinct subject sub-groups of differing strength levels (18 elite male athletes and 8 untrained male subjects, with the ISq conducted at a mean knee angle of 
$\left.118^{\circ}\right)$, however, reliability data were only reported for the untrained subjects $(\mathrm{CV}=4 \%$, ICC $=0.960)$ [31]. In a group of recreationally trained male subjects $(\geq 1$ year training experience), similar ICC values (0.97 and 0.99 at ISq $_{120}$ and ISq $_{90}$, respectively) were reported by Bazyler et al. (2015) [10]. Additionally, Drake et al. (2018) reported comparable $\mathrm{CV}$ and ICC values of $6 \%$ and 0.856 at $\mathrm{ISq}_{90}$ in a group of strength-trained males (mean (SD) training experience 4.1 (1.8) years) [11]. Moreover, Blazevich et al. (2002) reported an ICC of 0.97 in the ISq $q_{90}$ in a group of athletic men [16]. This suggests that training status, and by association maximal strength, may not influence ISq reliability. However, Palmer et al. (2017) reported CV and ICC values of $11.2 \%$ and 0.839 respectively at ISq ${ }_{120}$ as well as $12 \%$ and 0.885 at ISq $q_{90}$, respectively, in a group of resistance-trained females, which differs quite considerably from the rest of the available literature [17]. The reason for this is not readily apparent, though it may be related to the specific isometric squat apparatus used in this investigation [17]. The data reported here display similar levels of reliability and supports the observation that there is no relationship between maximal ISq strength and test-retest reliability of the ISq. This observation is consistent across different positions (e.g., $\mathrm{ISq}_{120}, \mathrm{ISq}_{90}, \mathrm{ISq}_{65}$ ), as reported here and elsewhere $[10,11,16,17,31]$.

The second objective of this study was to determine the reliability of PF measurement in the $\mathrm{ISq}_{65}$, compared to the ISq $\mathrm{I}_{120}$ and ISq $\mathrm{q}_{90}$, in a sub-sample of eligible subjects. To the authors' knowledge, this is the first study to investigate the use of an ISq position at a $65^{\circ}$ knee angle, which is somewhat surprising given it closely approximates the bottom position of the back squat (Hales et al., 2009; Swinton et al., 2012). All positions including ISq 65 displayed acceptable reliability based on ICC values (>0.8) [23-25]. We noted that, whilst it was reliable and not significantly different from ISq ${ }_{120}$ and ISq ${ }_{90}$ positions, the CV was highest at ISq 65 (Table 2). Furthermore, based on researcher observation and verbal feedback from the subjects, the ISq ${ }_{65}$ position was unfamiliar and as a result, subjects reported that it was more difficult to establish. Given the increased mobility demands of the ISq $\mathrm{q}_{65}$, some subjects had difficulty attaining the required positioning, which may have affected the reliability of the force output in this position. We speculate that in contrast with the ISq $q_{65}$ position, the ISq 120 and ISq $q_{90}$ positions require minimal skill and mobility, allowing subjects to focus their efforts on exerting maximal force into the bar. That said, it is also plausible that a greater number of familiarization sessions may have improved the reliability of the force output in the ISq $_{65}$ position [11] and, therefore, may be worth consideration for future research. Drake et al. (2018) observed improvements in PF in the $\mathrm{ISq}_{90}$ past three test days, indicating that more sessions may improve the reliability of the measure at each position [11].

TE values for each ISq position were calculated to indicate the change scores required over time to be considered real (Table 2). These values allow researchers and practitioners alike to determine what is the smallest change required over time to be considered real if they are working with subjects from the same population from which our sample was drawn. This is a valuable consideration for those using the ISq to monitor performance changes in response to training interventions. Based on our data, change scores of $10.5 \%, 14.2 \%$, and $11.0 \%$ are required to be considered real changes at $\mathrm{ISq}_{120}, \mathrm{Isq}_{90}$, and $\mathrm{ISq}_{65}$ respectively.

Overall, we demonstrate acceptable levels of reliability in an ISq test conducted at knee angles of $120^{\circ}$ and $90^{\circ}$, providing evidence that the ISq can be used to reliably measure PF. Additionally, we observed no relationship between the maximal strength of an individual and the reliability of ISq measurement. This is an important finding for sport science researchers and practitioners as it adds merit to the applicability of ISq measurement across athletes of different strength levels. For clinical research, the ISq may also have application as a measure of maximal strength in studies of sedentary/untrained subjects. For practitioners, the ISq offers a potential alternative to typical 1RM testing, which can be difficult to standardize and carries an inherent level of risk that may not be deemed acceptable. In addition, the data indicate that the ISq is a reliable test of maximal strength when conducted at a knee angle of $65^{\circ}$. However, it is important to note that this position 
is more difficult to attain and researchers may be best served by conducting the ISq at a $90^{\circ}$ or a $120^{\circ}$ knee angle, and this is perhaps the reason this data are more commonly reported in the literature.

Author Contributions: Conceptualization, A.E.L., R.W.D., T.L., P.M.J. and B.P.C.; methodology, A.E.L., R.W.D., P.M.J. and B.P.C.; formal analysis, A.E.L., R.W.D., P.M.J., J.M.A. and B.P.C.; investigation, A.E.L., R.W.D. and T.L.; data curation, A.E.L., R.W.D. and T.L.; writing-original draft preparation, A.E.L., R.W.D., T.L., P.M.J. and B.P.C.; writing-review and editing, A.E.L., R.W.D., P.M.J., J.M.A. and B.P.C. All authors have read and agreed to the published version of the manuscript.

Funding: This research received no external funding.

Institutional Review Board Statement: The study design, documentation and procedures were all approved by the University of Limerick Education and Health Sciences Research Ethics Committee, in accordance with the declaration of Helsinki (ethical approval number EHS_2016_12_09).

Informed Consent Statement: Informed consent was obtained from all subjects involved in the study.

Data Availability Statement: The data presented in this study are available on request from the corresponding author.

Acknowledgments: Authors acknowledge the contribution of research assistants Sorcha Holohan, Patrick Garvey, Jack Heavey and Niall Mulcahy as well as the subjects for their time and effort.

Conflicts of Interest: The authors declare no conflict of interest.

\section{References}

1. Abernethy, P.; Wilson, G.; Logan, P. Strength and Power Assessment. Sports Med. 1995, 19, 401-417. [CrossRef]

2. Baker, D.; Wilson, G.; Carlyon, B. Generality versus specificity: A comparison of dynamic and isometric measures of strength and speed-strength. Eur. J. Appl. Physiol. Occup. Physiol. 1994, 68, 350-355. [CrossRef]

3. Murphy, A.J.; Wilson, G.J. The ability of tests of muscular function to reflect training-induced changes in performance. J. Sports Sci. 1997, 15, 191-200. [CrossRef]

4. Sale, D.; Martin, J.; Moroz, D. Hypertrophy without increased isometric strength after weight training. Eur. J. Appl. Physiol. Occup. Physiol. 1992, 64, 51-55. [CrossRef]

5. Bazyler, C.D.; Sato, K.; Wassinger, C.; Lamont, H.S.; Stone, M.H. The efficacy of incorporating partial squats in maximal strength training. J. Strength Cond. Res. 2014, 28, 3024-3032. [CrossRef]

6. Nuzzo, J.L.; Taylor, J.L.; Gandevia, S.C. CORP: Measurement of upper and lower limb muscle strength and voluntary activation. J. Appl. Physiol. 2019, 126, 513-543. [CrossRef] [PubMed]

7. Arazi, H.; Asadi, A. One repetition maximum test increases serum indices of muscle damage and soreness in trained and untrained males. Apunts. Med. L'Esport 2013, 48, 49-54. [CrossRef]

8. Loturco, I.; Pereira, L.A.; Abad, C.C.; Gil, S.; Kitamura, K.; Kobal, R.; Nakamura, F.Y. Using bar velocity to predict maximum dynamic strength in the half-squat exercise. Int. J. Sports Physiol. Perform. 2016, 11, 697-700. [CrossRef] [PubMed]

9. Loturco, I.; Pereira, L.A.; Kobal, R.; Abad, C.C.; Rosseti, M.; Carpes, F.P.; Bishop, C. Do asymmetry scores influence speed and power performance in elite female soccer players? Biol. Sport 2019, 36, 209-216. [CrossRef] [PubMed]

10. Bazyler, C.; Beckham, G.; Sato, K. The Use of the Isometric Squat as a Measure of Strength and Explosiveness. J. Strength Cond. Res. 2015, 29, 1386-1392. [CrossRef] [PubMed]

11. Drake, D.; Kennedy, R.; Wallace, E. Familiarization, validity and smallest detectable difference of the isometric squat test in evaluating maximal strength. J. Sports Sci. 2018, 36, 2087-2095. [CrossRef]

12. Drake, D.; Kennedy, R.; Wallace, E. The validity and responsiveness of isometric lower body multi-joint tests of muscular strength: A systematic review. Sports Med. Open 2017, 3, 23. [CrossRef] [PubMed]

13. Brady, C.J.; Harrison, A.J.; Comyns, T.M. A review of the reliability of biomechanical variables produced during the isometric mid-thigh pull and isometric squat and the reporting of normative data. Sports Biomech. 2018, 19, 1-25. [CrossRef]

14. McLaughlin, T.; Dillman, C.; Gardner, T. A kinematic model of performance in the parallel squat by champion powerlifters. Med. Sci. Sports Exerc. 1977, 9, 128-133. [CrossRef]

15. Kompf, J.; Arandjelović, O. The sticking point in the bench press, the squat, and the deadlift: Similarities and differences, and their significance for research and practice. Sports Med. 2017, 47, 631-640. [CrossRef]

16. Blazevich, A.; Gill, N.; Newton, R. Reliability and Validity of Two Isometric Squat Tests. J. Strength Cond. Res. 2002, 16, 298-304. [PubMed]

17. Palmer, T.B.; Pineda, J.G.; Durham, R.M. Effects of knee position on the reliability and production of maximal and rapid strength characteristics during an isometric squat test. J. Appl. Biomech. 2017, 34, 111-117. [CrossRef] [PubMed]

18. Glassbrook, D.J.; Helms, E.R.; Brown, S.R.; Storey, A.G. A review of the biomechanical differences between the high-bar and low-bar back-squat. J. Strength Cond. Res. 2017, 31, 2618-2634. [CrossRef] 
19. Hales, M.E.; Johnson, B.F.; Johnson, J.T. Kinematic analysis of the powerlifting style squat and the conventional deadlift during competition: Is there a cross-over effect between lifts? J. Strength Cond. Res. 2009, 23, 2574-2580. [CrossRef]

20. International Powerlifting Federation Technical Rules Book 2021. Available online: https://www.powerlifting.sport/fileadmin/ ipf/data/rules/technical-rules/english/IPF_Technical_Rules_Book_2021docx.pdf (accessed on 20 July 2021).

21. Swinton, P.A.; Lloyd, R.; Keogh, J.W.; Agouris, I.; Stewart, A.D. A biomechanical comparison of the traditional squat, powerlifting squat, and box squat. J. Strength Cond. Res. 2012, 26, 1805-1816. [CrossRef]

22. Davies, R.W.; Carson, B.P.; Jakeman, P.M. Sex Differences in the Temporal Recovery of Neuromuscular Function Following Resistance Training in Resistance Trained Men and Women 18 to 35 Years. Front. Physiol. 2018, 9, 1480. [CrossRef]

23. Koo, T.K.; Li, M.Y. A guideline of selecting and reporting intraclass correlation coefficients for reliability research. J. Chiropr. Med. 2016, 15, 155-163. [CrossRef] [PubMed]

24. Atkinson, G.; Nevill, A.M. Statistical Methods For Assessing Measurement Error (Reliability) in Variables Relevant to Sports Medicine. Sports Med. 1998, 26, 217-238. [CrossRef]

25. Bland, J.M.; Altman, D.G. A note on the use of the intraclass correlation coefficient in the evaluation of agreement between two methods of measurement. Comput. Biol. Med. 1990, 20, 337-340. [CrossRef]

26. Cohen, J. Statistical Power Analysis for the Behavioral Sciences, 2nd ed.; Routledge: New York, NY, USA, 1988; pp. 9-13.

27. Hopkins, W.G. Measures of reliability in sports medicine and science. Sports Med. 2000, 30, 1-15. [CrossRef] [PubMed]

28. Ritti-Dias, R.M.; Avelar, A.; Salvador, E.P.; Cyrino, E.S. Influence of previous experience on resistance training on reliability of one-repetition maximum test. J. Strength Cond. Res. 2011, 25, 1418-1422. [CrossRef] [PubMed]

29. Benton, M.J.; Raab, S.; Waggener, G.T. Effect of training status on reliability of one repetition maximum testing in women. J. Strength Cond. Res. 2013, 27, 1885-1890. [CrossRef] [PubMed]

30. Beckham, G.K.; Sato, K.; Santana, H.A.; Mizuguchi, S.; Haff, G.G.; Stone, M.H. Effect of body position on force production during the isometric midthigh pull. J. Strength Cond. Res. 2018, 32, 48-56. [CrossRef]

31. Tillin, N.A.; Pain, M.T.G.; Folland, J. Explosive force production during isometric squats correlates with athletic performance in rugby union players. J. Sports Sci. 2013, 31, 66-76. [CrossRef] 